\title{
Appendiceal perforation due to biliary stent migration in a neutropenic patient with lung cancer receiving chemotherapy: A case report
}

\author{
PASI PENGERMÄ ${ }^{1,2}$, JEVGENI KATUNIN ${ }^{1}$, ARTO TURUNEN ${ }^{1}$, PIERPAOLO SILERI ${ }^{3}$, \\ GABRIELLA GIARRATANO $^{4,5}$, ARI PALOMÄKI ${ }^{6}$ and ARISTOTELIS KECHAGIAS ${ }^{1}$ \\ ${ }^{1}$ Department of Gastrointestinal Surgery, Kanta-Häme Central Hospital, Hämeenlinna 13530; \\ ${ }^{2}$ Department of Gastrointestinal Surgery, Kuopio University Hospital, Kuopio 70210, Finland; \\ ${ }^{3}$ Department of Surgery, Università Vita Salute San Raffaele, Milan I-20132; ${ }^{4}$ Department of General Surgery, \\ Casa di Cura Nuova Itor, Rome I-00158; ${ }^{5}$ Department of General Surgery, University of Rome Tor Vergata, \\ Rome I-00133, Italy; ${ }^{6}$ Department of Emergency Medicine, Kanta-Häme Central Hospital, Hämeenlinna 13530, Finland
}

Received February 25, 2020; Accepted January 21, 2021

DOI: $10.3892 / \mathrm{mco} .2021 .2298$

\begin{abstract}
The use of biliary stents has become a common and usually safe procedure. However, the migration of biliary stents is an uncommon but well-recognized event after endoscopic retrograde cholangiopancreatography. The migration of plastic stents usually does not result in complications and are spontaneously eliminated from the gastro-intestinal tract. Additionally, $<1 \%$ of migrated stents result in intestinal perforation, which typically occurs at the duodenum. Chemotherapeutic agents may cause gastrointestinal toxicity and hematologic toxicity predisposing to neutropenic enterocolitis. The current study reports a patient with an unprecedented case of biliary stent migration resulting in appendiceal gangrene and perforation in a neutropenic patient under chemotherapy for metastatic small cell lung cancer.
\end{abstract}

\section{Introduction}

Chemotherapy for cancer is associated with a constellation of adverse effects including toxicity in the gastro-intestinal tract. Cytotoxic drugs may cause significant injury and disruption of the gut mucosa with manifestations of varying severity such as diarrhea or bacterial translocation leading to local or disseminated infection (1). Patients with chemotherapy-induced immunosuppression may present with neutropenic colitis which can be a life-threatening condition particularly in the

Correspondence to: Mr. Pasi Pengermä, Department of Gastrointestinal Surgery, Kanta-Häme Central Hospital, Ahvenistontie 20, Hämeenlinna 13530, Finland E-mail: pasi.pengerma@gmail.com

Key words: appendiceal perforation, biliary stent, stent migration, neutropenic enterocolitis, topotecan less common occasion of full-thickness bowel necrosis and perforation (2).

Acute complications are very common in patients receiving chemotherapy. Due to significant co-morbidity or various concomitant parameters they may constitute challenging inter-disciplinary cases at the emergency department. Our objective is to present and discuss an unprecedented case of appendiceal perforation and gangrene in a patient with metastatic small-cell lung cancer and chemotherapy-induced neutropenic enterocolitis.

\section{Case report}

In September 2018 a 66-year-old woman with multiple co-morbidity presented at the emergency department of our hospital with a worsening abdominal pain of four days duration. The patient was under chemotherapy with topotecan since June 2018 for a primarily inoperable small-cell lung carcinoma with bone metastases. The last dose of topotecan was administered two weeks previously. Earlier chemotherapy regimens included carboplatin and etoposide, first started in November 2017. Her comorbidities included coronary artery disease, insulin-dependent type II diabetes, hypertension, hypercholesterolemia, and liver steatosis. Acetylsalicylic acid was used as an anti-aggregant medication. The patient had exocrine pancreatic insufficiency and an established diagnosis of chronic pancreatitis since 2008. Thereafter she suffered from several episodes of acute-on-chronic pancreatitis with the last episode in December 2017. In July 2018 she underwent an endoscopic retrograde cholangiopancreatography (ERCP) in order to relieve distal biliary obstruction caused by a chronic pancreatic pseudocyst in the pancreatic head. At this procedure a sphincterotomy of the sphincter of Oddi was performed and a 5-cm long plastic biliary stent of 10 -french diameter was successfully deployed in the common bile duct. In addition, the surgical anamnesis comprised an open cholecystectomy in 2003. 
The initial clinical examination revealed guarding on the right lower quadrant. The last bowel movement occurred four days earlier. The patient had fever and mild hypotension. The laboratory results showed leukocytopenia (white blood cell count=1,7×10E9/1), neutropenia (neutrophil count $=0,74 \times 10 \mathrm{E} 9 / 1$ ), profuse thrombocytopenia (platelet count $=9 \times 10 E 9 / 1)$, normocytic anemia (hemoglobin=92 g/l), whereas the c-reactive protein (crp) was high at $264 \mathrm{mg} / \mathrm{l}$. The transaminase, bilirubin, glomerular filtration rate, and amylase levels were normal and the alkaline phosphatase level was slightly increased at a chronic basis (164 U/1) consistent to the presence of the stent and to the medication. Blood cultures were negative. A computed tomography (CT) -scan of the abdomen showed that the biliary stent had migrated into the lumen of the appendix (Figs. 1A and B, and 2). The lumen was obstructed and the appendix thickened due to inflammation (Fig. 1B). There was also peri-appendiceal fat stranding without any extra-luminal air.

Immediate resuscitation with fluids and broad-spectrum antibiotics (piperacillin/tazobactam) were initiated and hemodynamic stability was restored. The patient was transfused with platelets and a leukocyte-stimulating factor (filgrastim) and corticosteroids were started. After transfusions the platelet count increased to $80 \times 10 \mathrm{E} 9 / 1$. A colonoscopy was attempted a few hours after admission with the intent to relieve the appendix by removing the stent and to continue with antibiotic therapy for the inflamed appendix (unless deterioration would occur). The endoscopy was abandoned due to poor visibility and an exploratory laparotomy was performed using a $7-\mathrm{cm}$ incision at the McBurney point. Laparoscopic surgery was not performed due to high anesthesiologic risk. Appendiceal inflammation was confirmed. The stent had perforated the appendix at its apex with half of the stent being situated free in the peritoneal cavity whereas the other half was inside the appendix clogging its lumen. The wall of the caecum appeared inflamed but there were no findings of frank peritonitis. The stent was removed and appendicectomy was performed with local lavage and drain placement.

The patient's immediate post-operative course was uneventful. The crp reached a peak on the second postoperative day $(431 \mathrm{mg} / \mathrm{l})$ and then it gradually dropped to $55 \mathrm{mg} / \mathrm{l}$ at discharge. The drain was removed on the third postoperative day. The leukocyte-stimulating factor was discontinued as the white blood cell count was restored. She was discharged nine days after the operation. Antibiotic therapy with cephalexin continued for seven more days. The pathologic examination of the removed appendix showed gangrenous changes with perforation and no neoplasia (Fig. 3).

Ten days after discharge she returned to the emergency department due to abdominal pain and fever. There was local guarding at the right lower quadrant and the crp was $27 \mathrm{mg} / \mathrm{l}$. A CT-scan revealed a $2 \times 3$-centimeter abscess located pre-sacrally and medially to the caecum. The patient was treated with broad-spectrum antibiotics alone (piperacillin/tazobactam) as the location of the abscess precluded percutaneous drainage. Subsequent CT-imaging showed near-total elimination of the infection. She was transferred to a health center after 11 days of hospitalization where a 5-day course of oral levofloxacin was continued.
The patient consented in written for the publication of her case study.

\section{Discussion}

Biliary stent migration is a rather uncommon event and bowel perforation due to the migrated stent is extremely rare (3). Migrated plastic stents in particular are normally eliminated from the gastro-intestinal tract without any consequence (3). The most common site of a perforation due to a stent is the duodenum (4-6). Sporadic cases of small- or large- bowel perforation have been reported $(7,8)$. Interestingly, such perforations have occurred in conjunction with a separate predisposing factor such as diverticula, adhesions, or incarcerated bowel in an incisional hernia $(7,8)$. To the best of our knowledge stent migration into the appendix with perforation has not been reported previously.

Various cytotoxic agents may cause colitis of varying extent (1). In general, neutropenic enterocolitis should be suspected in immunosuppressed patients with abdominal pain, especially those receiving chemotherapy (9). Typical features in patients with neutropenic enterocolitis comprise a low neutrophil count, fever, and abdominal pain due to inflammation with bowel wall thickening of the ileocolonic region $(2,9,10)$. The histopathological examination of specimens with neutropenic enterocolitis may include multiple alterations such as areas of patchy necrosis, mucosal hemorrhage, ulceration, edema, perforation, infiltrating organisms, and typically, depletion of inflammatory cells (9).

The patient of our case was under topotecan treatment which is a topoisomerase inhibitor employed as a second-line chemotherapeutic agent for small-cell lung cancer (10). A recent series of 64 patients with small-cell lung cancer who received 177 cycles of topotecan reported significant hematologic toxicity including $25 \%$ of neutropenia, $11 \%$ of thrombocytopenia, and $20.3 \%$ of grade 3 or 4 anemia (10). The greater majority $(80 \%)$ of hospitalizations were due to febrile neutropenia which occurred in $17 \%$ of the patients (10). Although histopathology is the gold standard for the final verification of neutropenic enterocolitis, in real-life clinical settings diagnosis and decisions are based on clinical, laboratory, and radiologic findings $(2,9,10)$. Diagnostic colonoscopy for mucosa inspection and biopsy acquisition should be used very selectively - or even avoided - in patients with clinical suspicion of neutropenic colitis as gas insufflation may increase the risk of free perforation due to bowel wall alterations and increased friability $(2,9)$.

Acute appendicitis is part of the differential diagnosis of neutropenic enterocolitis (2), however both entities were concurrently present in the patient reported here. In this case it is still challenging to discern a single factor as the cause of the gangrenous changes in the appendix. Neutropenia may cause numerous areas of mucosal patchy necrosis with bowel wall friability, but it is rarely the reason of full-thickness necrosis or free perforation $(2,9)$. Indeed, conservative treatment with aggressive resuscitation displays high-success rates and currently surgery is reserved for more complicated cases (2). In our patient, it is very likely that patchy ischemia in the surface of the appendiceal mucosa was triggered by the neutropenic status and that further deterioration leading to perforation 

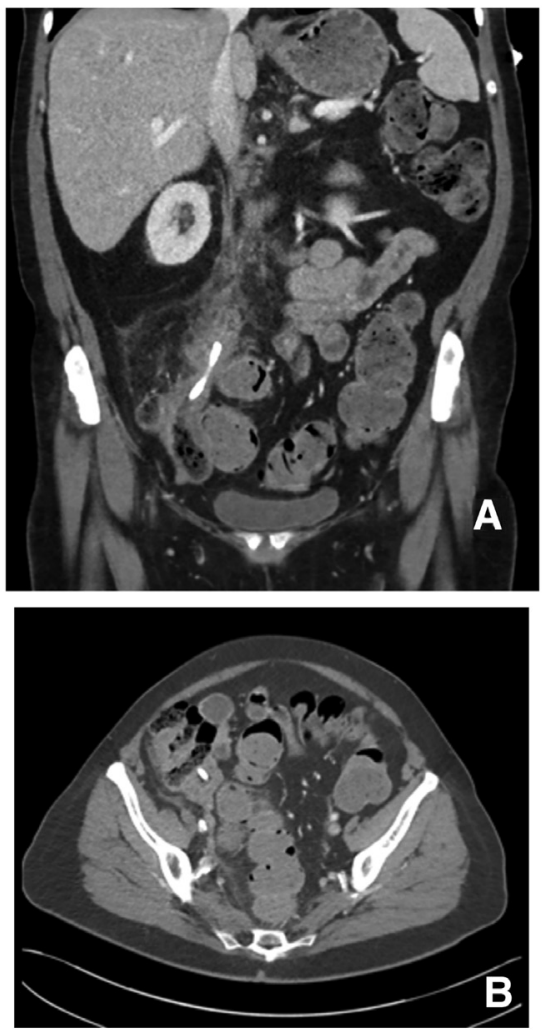

Figure 1. (A) Computed-tomography abdominal imaging revealed the intra-appendiceal location of the stent with lumen occlusion. The appendix was severely thickened due to inflammation with peri-appendiceal fat stranding. (B) Location of the stent in axial view.

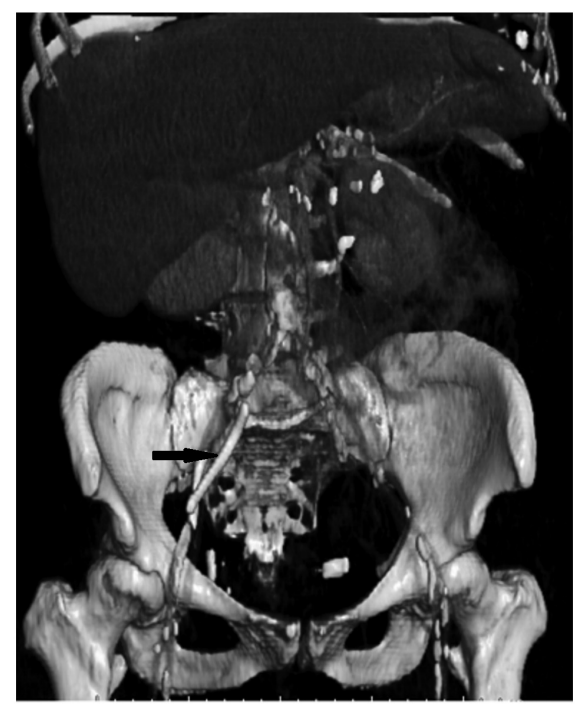

Figure 2. Location of the migrated stent at the right lower abdomen with 3-dimensional reconstruction of the computed-tomography data.

ensued due to the increased pressure caused by the obstruction of the lumen from the stent. Moreover, the relatively reduced number of neutrophils in the appendix of this patient possibly favored friability and bacterial growth.

Our patient had high surgical- and anesthesiologic- risk by reason of the remarkable hematologic toxicity and comorbidities. The initial therapeutic strategy was to assess the feasibility of a short trial of conservative therapy with broad-spectrum

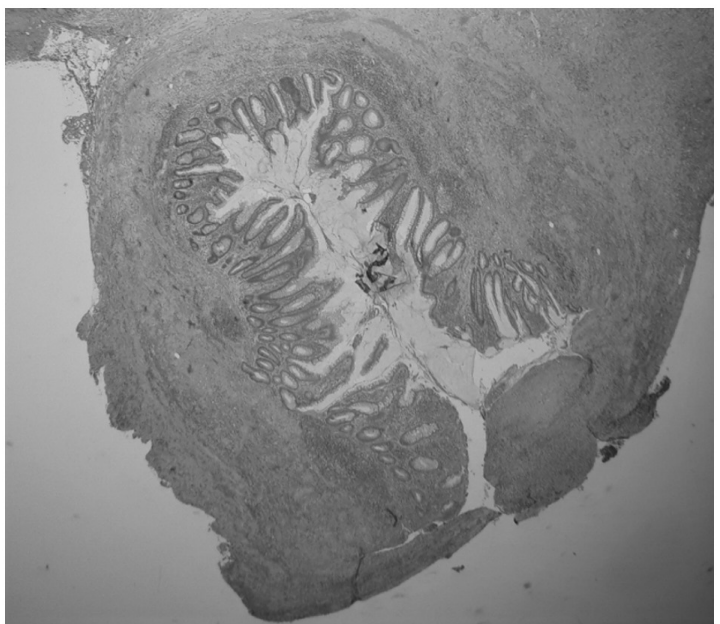

Figure 3. Microscopic view of the surgical specimen. Mucosal and submucosal hemorrhage with superficial mucosal necrosis and perforation at the tip of the appendix is demonstrated (black arrow). The presence of white blood cells was markedly reduced compared with common appendicitis' specimens in patients without hematologic toxicity.

antibiotics, fluid resuscitation, correction of the hematologic parameters, and relieving the appendix with endoscopic removal of the stent. By judging the herein experience in retrospect, primary surgery with appendicectomy (after aggressive transfusions to restore the platelet count) would be suggestable due to the high risk of gangrenous changes and perforation in the ground of neutropenia, sepsis, and appendiceal-lumen obstruction from a migrated biliary stent.

In conclusion, migration of a plastic biliary stent in the gastro-intestinal tract is almost always innocuous but an increased risk of perforation may exist in the case of concomitant neutropenic enterocolitis. A CT-scan should always be obtained in such patients in order to assess the position of the stent and to evaluate signs of perforation. The combination of stent migration into the appendix and neutropenia may itself be highly suggestive of appendiceal ischemia and necrosis, despite the absence of perforation in imaging. Primary treatment option should be urgent appendicectomy after aggressive optimization of the hematologic status.

\section{Acknowledgements}

The authors would like to acknowledge to Dr Johanna Saukkonen (Department of Radiology, Kanta-Häme Central Hospital) for reconstructing the 3-dimensional image that illustrated the location of the migrated stent. The authors would also like to thank Pathologists Mr. Kimmo Lähteenmäki and Ms. Marita Laurila (both, Department of Pathology, Kanta-Häme Central Hospital) for the histological images of the surgical specimen.

\section{Funding}

No funding was received.

\section{Availability of data and materials}

The datasets used and/or analyzed during the present study are available from the corresponding author on reasonable request. 


\section{Authors' contributions}

PP, JK, AT, PS, GG, AP and AK conceived and designed the current study. PP, JK and AK acquired the data. PP, JK, AT, PS, $\mathrm{GG}, \mathrm{AP}$ and AK analyzed and interpreted the data. PP, JK, AT, PS, GG, AP and AK wrote, revised and edited the manuscript. All authors read and approved the final manuscript.

\section{Ethics approval and consent to participate}

Not applicable.

\section{Patient consent for publication}

The patient provided their written consent for the publication of their data and associated images.

\section{Competing interests}

The authors declare that they have no competing interests.

\section{References}

1. Boussios S, Pentheroudakis G, Katsanos K and Pavlidis N: Systemic treatment-induced gastrointestinal toxicity: Incidence, clinical presentation and management. Ann Gastroenterol 25: 106-118, 2012.
2. Rodrigues FG, Dasilva G and Wexner SD: Neutropenic enterocolitis. World J Gastroenterol 23: 42-47, 2017.

3. Dumonceau JM, Tringali A, Blero D, Deviere J, Laugiers R, Heresbach D, Costamagna G; European Society of Gastrointestinal Endoscopy: Biliary stenting: Indications, choice of stents and results: European society of gastrointestinal endoscopy (ESGE) clinical guideline. Endoscopy 44: 277-298, 2012.

4. Malgras B, Pierret C, Tourtier JP, Olagui G, Nizou C and Duverger V: Double sigmoid colon perforation due to migration of a biliary stent. J Visc Surg 148: e397-e399, 2011.

5. Belyaev O, Müller CA and Uhl W: Double sigmoid colon perforation by a migrated biliary stent. Acta Chir Belg 108: 125-126, 2008.

6. Anderson EM, Phillips-Hughes J and Chapman R: Sigmoid colonic perforation and pelvic abscess complicating biliary stent migration. Abdom Imaging 32: 317-319, 2007.

7. Mady RF, Niaz OS and Assal MM: Migrated biliary stent causing perforation of sigmoid colon and pelvic abscess. BMJ Case Rep 13: bcr2014206805, 2015.

8. Yilmaz Ö, Kiziltan R, Aydin O, Bayrak V and Kotan Ç: A rare complication of biliary stent migration: Small bowel perforation in a patient with incisional hernia. Case Rep Surg 2015: 860286, 2015.

9. Xia R and Zhang X: Neutropenic enterocolitis: A clinico-pathological review. World J Gastrointest Pathophysiol 10: 36-41, 2019.

10. Popovic F, Jakopovic M, Samarzija M, Cucevic B, Kukulj S, Rogliç M and Plestina S: P1.07-013 treatment related side effects of oral topotecan in small cell lung cancer: Topic: Drug treatment alone and in combination with radiotherapy. J Thorac Oncol 12: S703, 2017. 